\title{
OLD AND NEW IN MÖBIUS GROUPS
}

\author{
LARS V. AHLFORS*
}

\section{Introduction}

In recent years there has been strong activity in the area of Möbius transformations in several dimensions. It all goes back to Poincaré who was the first to observe that the action of the Möbius group in the complex plane can be lifted to the surrounding three-space, and specifically to the upper half-space which he recognized as a model of three-dimensional noneuclidean geometry. At that time there was no serious interest in more than three dimensions.

In due course the advances in Fuchsian groups and automorhpic functions provided a natural stepping stone to higher dimensions. Among the pioneers was Eberhard Hopf who generalized the ergodic theory of Fuchsian groups to $n$ dimensions as early as in the 1930s. A more recent factor was the increasing interest in Kleinian groups and Teichmüller spaces. The problems with the limit set of a Kleinian group seemed to indicate that the methods based on conformal and quasiconformal mappings in the plane were insufficient, and that something new was needed. Although many problems remain unsolved there is little doubt that the passage to $n$ dimensions has thrown light also on the classical case.

Progress has been made in several directions, but nothing has given a greater impulse to the study of Möbius groups in several dimensions than the so-called rigidity theorem of G. D. Mostow [Mo], published in 1968. Several rigidity theorems for discontinuous groups were already known, but it was a complete surprise that something as simple as Mostow's theorem would be valid for Möbius groups.

My original plan for this talk was to make it a survey of the state of the art in Möbius groups, but I soon realized that this would be impossible. Too much has been done to be covered even superficially in a single lecture, and so much exists only in preprints, not all available to me, that it is even difficult to know what has been done and what not. Also, there are almost no expository papers, partly because the leading journals are reluctant to accept them, and the research papers are mostly so

* Research supported by the National Science Foundation.

Lecture given in the $11^{\text {th }}$ Rolf Nevanlinna Colloquium at Jyväskylä August 10-13. 1983. 
complex that it takes years to study them adequately. I was therefore pleased to learn that Acta Mathematica has accepted a very readable paper by Stephen Agard which reviews what is known but unpublished and goes on to present a new and entirely elementary approach to Mostow rigidity. Accordingly, I decided to devote a major part of my talk to what I have learned from Agard's paper. This may be unfair to others, but I had to make a choice.

The rest of the talk will deal with a question that has occupied me lately, namely the use of Clifford numbers in connection with Möbius transformations. Part of this theory is very old, and practically forgotten, part is fairly new, but deserves to be better known. I believe a revival of interest would be very timely.

\section{Basic notions}

One of the difficulties when speaking about Möbius groups is that terminology and notations have not been standardized; everybody uses his own, and I shall have to do the same. Because the ideas are easy to recognize it is never difficult to translate from one language to another, but it is unquestionably a nuisance.

The basic Möbius group is the one that acts on $R^{n}$, or more precisely on $\bar{R}^{n}=$ $R^{n} \cup\{\infty\}$. I shall denote it by $\mathrm{M}\left(R^{n}\right)$ with an explicit warning that it is not restricted to sense-preserving mappings. By definition, $g \in \mathrm{M}\left(R^{n}\right)$ if $g: \bar{R}^{n} \rightarrow \bar{R}^{n}$ is a homeomorphism whose derivative $g^{\prime}(x)$ at each $x \neq \infty$ and $g^{-1}(\infty)$ is a conformal matrix, i.e. a positive multiple of a matrix in $\mathrm{O}(n)$. In analogy with the distinction between $\mathrm{O}(n)$ and $\mathrm{SO}(n)$ it would be natural to let $\mathrm{SM}\left(R^{n}\right)$ refer to the subgroup of sensepreserving mappings, but it will not be needed in this paper.

The subgroup that keeps the upper half space $U^{n}$ invariant will be denoted by $\mathrm{M}\left(U^{n}\right)$; the corresponding subgroup for the unit ball $B^{n}$ is called $\mathrm{M}\left(B^{n}\right)$. The boundary $\partial U^{n}$ is identified with $R^{n-1}$, and $\partial B^{n}$ is the unit sphere $S^{n-1}$.

We shall use the standard notations $x=\left(x_{1}, \ldots, x_{n}\right) \in R^{n}$ and $x y=(x, y)=x_{1} y_{1}+\ldots$ $\ldots+x_{n} y_{n},(x, x)=|x|^{2} . \mathrm{M}\left(R^{n}\right)$ is generated by all translations $x \rightarrow x+b, b \in R^{n}$, dilations $x \rightarrow \varrho x, \varrho>0$, and the inversion $x \rightarrow \sigma(x)=x /|x|^{2}$. For practical reasons it is convenient to include the isometries $x \rightarrow k x, k \in \mathrm{O}(n)$, among the generators.

I prefer to use $I_{n}$ (or $I$ ) for the unit matrix (identity), and I have the personal habit of writing $Q(x)$ for the matrix with elements $x_{i} x_{j} /|x|^{2}$. In this notation the derivative of $\sigma(x)$ is $\sigma^{\prime}(x)=|x|^{-2}(I-2 Q(x))$. Note that $(I-2 Q(x))^{2}=I$ and $I-2 Q(x) \in \mathrm{O}(n)$.

By comparison, Agard [Ag 1,2] uses id for the identity, $t_{b}$ for translation by $b$, and $h_{\varrho}$ for dilation by $\varrho$. There are some advantages to the use of letters for mappings, but on the whole I find formulas which contain the variable, usually $x$, much easier to read. I have accepted his $\sigma$ for inversion, which I hope will stay, but for typographical reasons I am apt to use $x^{*}$ as an alternate notation. For instance, $\left(x^{*}-y^{*}\right)^{*}$ would be rather awkward in Agard's notation. 
At the time of the Nevanlinna Colloquium I had not yet seen A. Beardon's new book on The Geometry of Discrete Groups [Be], a most welcome addition to the literature of the subject. If I had, it would undoubtedly have influenced my exposition in important ways.

\section{The matrix representation}

In the case of complex Möbius transformations it is natural and easy to express $\mathrm{M}(C)$ by $\mathrm{GL}_{2}(C)$ or $\mathrm{SL}_{2}(C)$ reduced modulo $C^{*}=C \backslash\{0\}$ or $\left\{ \pm I_{2}\right\}$. For $n>2$ this matrix representation is not available, but it is almost obvious that the groups $\mathrm{M}\left(R^{n-1}\right), \mathrm{M}\left(U^{n}\right)$ and $\mathrm{M}\left(B^{n}\right)$ are mutually isomorphic. In connection with the projective theory of hyperbolic geometry developed by Cayley and Klein it also became clear that these Möbius groups are isomorphic with a subgroup of the orthogonal group $\mathrm{O}(n, 1)$ associated with the quadratic form $x_{1}^{2}+\ldots+x_{n}^{2}-x_{n+1}^{2}$.

Personally, I must admit that I have been reluctant to make use of this isomorphism, mainly because the step from $\mathrm{M}\left(R^{n-1}\right)$ to $\mathrm{O}(n, 1)$ requires two more dimensions and, as it seemed to me, a disproportionate complication of the formulas. For instance, to replace $\mathrm{M}(C)$ by $\mathrm{O}(3,1)$ makes little sense. I now realize that my judgment may have been a little too rash, for when I took a second look I found that the situation is much simpler and more manageable than I expected.

In my opinion the best approach is to emphasize the analogy with stereographic projection. Let me start from the identity

$$
\left(\frac{1+|x|^{2}}{2}\right)^{2}=\left(\frac{1-|x|^{2}}{2}\right)^{2}+|x|^{2}
$$

where $x=\left(x_{1}, \ldots, x_{n}\right) \in R^{n}$. It can be rewritten either as

or as

$$
\left|\frac{2 x}{1+|x|^{2}}\right|^{2}+\left(\frac{1-|x|^{2}}{1+|x|^{2}}\right)^{2}=1
$$

$$
\left(\frac{1+|x|^{2}}{1-|x|^{2}}\right)^{2}-\left|\frac{2 x}{1-|x|^{2}}\right|^{2}=1 \text {. }
$$

Formula (3.1) shows that

$$
\left(y, y_{n+1}\right)=\left(\frac{2 x}{1+|x|^{2}}, \frac{1-|x|^{2}}{1+|x|^{2}}\right) \in S^{n}
$$

and one recognizes the familiar form of the stereographic projection from $R^{n}$ to $S^{n}$, except that the upper and lower hemispheres have been interchanged. Similarly, by (3.2)

$$
\left(y, y_{n+1}\right)=\left(\frac{2 x}{1-|x|^{2}}, \frac{1+|x|^{2}}{1-|x|^{2}}\right) \in S_{h}^{n}
$$


where $S_{h}^{n}$ is the hyperboloid $y_{n+1}^{2}-\sum_{i=1}^{n} y_{i}^{2}=1$. $S_{h}^{n}$ can be regarded as the hyperbolic counterpart of $S^{n}$. Observe that we have chosen the sign of $y_{n+1}$ so that $B^{n}$ is mapped on the part of $S_{h}^{n}$ in $U^{n+1}$ with 0 being mapped on $(0,1)$.

The mapping $B^{n} \rightarrow S_{h}^{n}$ is conformal provided that $S_{h}^{n}$ is equipped with the metric $d s_{h}^{2}=d y_{n+1}^{2}-\sum_{i=1}^{n} d y_{i}^{2}$. Indeed, one verifies that $d s_{h}^{2}=4|d x|^{2} /\left(1-|x|^{2}\right)^{2}$ which makes the mapping an isometry from the hyperbolic metric of $B^{n}$ to $d s_{h}$. It follows that $\mathrm{M}\left(B^{n}\right)$ is isomorphic to the matrix group $\mathrm{SO}(n, 1)^{+}$which preserves the metric $d s_{h}$ and the sign of $y_{n+1}$.

I wish to be more explicit. As a standard conformal mapping of $B^{n}$ on itself which carries $a \in B^{n}$ to 0 I have advocated the use of the mapping

$$
x \rightarrow T_{a}(x)=\frac{\left(1-|a|^{2}\right) x-\left(1+|x|^{2}-2 a x\right) a}{1-2 a x+|x|^{2}|a|^{2}}
$$

which is the analog of the complex mapping $z \rightarrow(z-a) /(1-\bar{a} z)$. The most general conformal self-mapping of $B^{n}$ is $x \rightarrow k T_{a}(x)$ with $k \in \mathrm{O}(n)$.

One finds, without too much computation, that the isometry between $\mathrm{M}\left(B^{n}\right)$ and $\mathrm{SO}(n, 1)^{+}$makes the mapping $T_{a}$ correspond to the matrix

$$
T_{a}=I_{n+1}+\frac{2}{1-|a|^{2}}\left(\begin{array}{cc}
a a^{T} & -a \\
-a^{T} & |a|^{2}
\end{array}\right) .
$$

In this matrix $a$ appears as a vertical vector with $a^{T}$ as its horizontal transpose, while $a a^{T}$ is the $n \times n$ matrix $\left\|a_{i} a_{j}\right\|$. The mapping $k T_{a}$ corresponds to $\left(\begin{array}{ll}k & 0 \\ 0 & 1\end{array}\right) T_{a}$ with $k \in \mathrm{O}(n)$. The relatively simple form of these matrices is an encouraging sign.

My belated insight in these matters was prompted by reading N. J. Wielenberg's thesis [Wi].

\section{Discrete subgroups}

Before dealing with rigidity we shall assemble a few facts about discrete subgroups. Let $\Gamma$ be a discrete subgroup of either $\mathrm{M}\left(U^{n}\right)$ or $\mathrm{M}\left(B^{n}\right)$; we shall feel free to move from one to the other without further comment. It acts discontinuously on $U^{n}$ or $B^{n}$, and there exists a fundamental polyhedron $P(\Gamma)$ representing the quotient space $U^{n} / \Gamma$ or $B^{n} / \Gamma$. We shall disregard the fact that the quotient space need not be a manifold in the strict sense.

If $\Gamma$ is infinite, as we shall assume, the orbits $\Gamma x$ converge to the limit set $\Lambda$ on the boundary $R^{n-1}$ or $S^{n-1}$. We consider only the case of non-elementary groups with more than two and hence infinitely many limit points. The set $\Lambda$ has also been called the topological limit set and denoted by $\Lambda_{T}$. This is to distinguish it from the conical limit set $\Lambda_{C}$ and the horocyclical limit set $\Lambda_{H}$. These names refer to the ways the orbits approach the limit points, but the precise definitions are not very relevant for this exposition. We note only that $\Lambda_{T} \supset \Lambda_{C} \supset \Lambda_{H}$. 
$\Gamma$ is said to be of the first kind if $\Lambda=R^{n-1}$ or $S^{n-1}$, of the second kind if not: in the latter case $\Lambda$ is a closed, nowhere dense subset of the boundary. Discrete subgroups are also classified according to the convergence or divergence of a series connected with the orbits; for $B^{n}$ it is the series

$$
\sum_{\gamma \in \Gamma}(1-|\gamma x|)^{n-1},
$$

convergence or divergence being the same for all $x \in B^{n}$. A group of the second kind is always of convergence type. More important, $\Gamma$ is of convergence type if and only if $B^{n} / \Gamma$ has a Green's function for the class of harmonic functions with respect to the hyperbolic metric of $B^{n}$. In other words, $\Gamma$ is of divergence type if and only if $B^{n} / \Gamma \epsilon$ $\mathrm{O}_{G}$. Because $\mathrm{O}_{G} \subset \mathrm{O}_{H B}$ (no bounded harmonic functions other than constants) it follows that any $\Gamma$ of divergence type acts ergodically on $S^{n-1}$, i.e. every measurable $\Gamma$-invariant subset of $S^{n-1}$ has either zero measure or the full measure of $S^{n-1}$.

Traditionally, groups with finite co-volume have played an important role. These are the groups whose fundamental polyhedron has finite noneuclidean volume, and it is to these groups that Mostow's original theorem applies. Tsuji [Ts] has proved that all groups with finite co-volume are of divergence type, while the converse is not true. His proof is for $B^{2}$, but the generalization to arbitrary $B^{n}$ is immediate.

D. Sullivan [Su] and Agard prove Mostow's result under the weaker hypothesis of divergence type. Agard's proof is elementary, although not easy. Sullivan makes essential use of ergodic theory and his style makes it difficult to follow all details. The proofs are very different, and they are both important contributions to the present state of knowledge.

\section{Mostow rigidity}

5.1. We come now to the topic of Mostow rigidity. I shall try to describe how this whole complex of problems is viewed by S. Agard in his paper for Acta Mathematica entitled "A geometric proof of Mostow's rigidity theorem for groups of divergence type". Naturally, I shall be able to give only a general outline of the underlying ideas.

The basic situation is as follows: Let $\Gamma$ be a discrete subgroup of $\mathrm{M}\left(U^{n+1}\right)$. A homeomorphism $f: U^{n+1} \rightarrow U^{n+1}$ is said to be compatible with $\Gamma$ if $f \gamma f^{-1}$ is a Möbius map for all $\gamma \in \Gamma$. Typically, this would be the case if $\Gamma^{\prime}$ is another discrete subgroup and $f$ arises by lifting a map $U^{n+1} / \Gamma \rightarrow U^{n+1} / \Gamma^{\prime}$ to $U^{n+1}$. In his original paper Mostow considers only the case $n \geqq 2$. He assumes that $\Gamma$ has finite co-volume, and he proves that if $f$ is quasiconformal and compatible with $\Gamma$, then $f$ extends to a conformal mapping $\bar{f}$ of $R^{n}$ so that $\Gamma$ and $\bar{f} \Gamma \bar{f}^{-1}$ are conjugate subgroups of $\mathrm{M}\left(R^{n}\right)$. According to a terminology introduced by Sullivan $\Gamma$ is said to be Mostow-rigid as soon as compatibility forces $\bar{f}$ to be conformal.

For $n \geqq 2$ a quasiconformal mapping of $U^{n+1}$ extends to a quasiconformal mapping of the boundary $R^{n}$, and this plays a crucial role in Mostow's proof. For 
$n=1$ this is no longer true, but the boundary mapping is still quasisymmetric on $R^{1}$. In a later paper Mostow has shown for this case, that $f$ is either Möbius or totally singular on $R^{1}$, in a strong sense. This question has also been analyzed by T. Kuusalo [Ku], but according to Agard the exact sense in which Mostow's result is generally valid remains to be clarified.

5.2. As indicated Sullivan and Agard both prove Mostow rigidity under the weaker hypothesis of divergence type. I have been particularly impressed by Agard's proof because of its completely elementary nature. It is rather long, but only because he includes all details and proves more than he needs. The main feature of the proof is that it does not use any ergodic theory, even in the comparatively unsophisticated sense of E. Hopf.

The novelty in Agard's proof is that he has recognized a strong connection between rigidity and certain half forgotten denseness theorems. The strongest previously known result of this kind is in P. J. Myrberg's paper of 1931 [My]. It has always been recognized that this was an important paper, but few have had the tenacity to study it in detail. The original paper dealt only with a special class of Fuchsian groups, but Agard shows that Myrberg's result is valid even in the most general case.

5.3. Agard's starting point is an innocent looking lemma which nevertheless has strong consequences. I shall refer to it as

Step I. Suppose that $g \in \mathrm{GL}(n)$ and that $\gamma$ and $g \gamma g^{-1}$ are both in $\mathrm{M}\left(R^{n}\right)$. If, in addition, $\gamma(\infty) \neq \infty$, then $g$ is a conformal matrix.

The proof consists in showing that the symmetrized matrix $g^{T} g$ has only one eigen-value (as before, $g^{T}$ is the transpose of $g$ ). It could be condensed to about half a page, but Agard chooses a broader exposition.

5.4. In order to explain the idea of denseness I shall introduce some standard notations, not exactly the same as Agard's. Let $p$ and $q$ be distinct points in $R^{n}$. The geodesic in $U^{n+1}$ which joins $p$ to $q$ will be denoted by $l(p, q)$. As a standard Möbius transformation which maps $l(p, q)$ on $l(0, \infty)$ we choose

$$
l_{p q}(x)=\left(x^{*}-q^{*}\right)^{*}-\left(p^{*}-q^{*}\right)^{*}
$$

which tends to the identity for $p \rightarrow 0, q \rightarrow \infty$ (recall that $x^{*}=\sigma(x)=x /|x|^{2}$ ). All transformations with $p$ as attractive and $q$ as repellent fixed point are of the form

$$
l_{p q}^{-1} \varrho k l_{p q}
$$

with $0<\varrho<1, k \in O(n)$. Agard denotes the whole set of mappings (5.2) by $\Lambda_{p q}$.

Instead of considering mappings $f: U^{n+1} \rightarrow U^{n+1}$ Agard focuses the attention on homeomorphisms $f: \bar{R}^{n} \rightarrow \bar{R}^{n}$ which are compatible with $\Gamma$ on $R^{n}$. For the condition of finite co-volume or divergence type it is of course still necessary to extend $\Gamma$ to $U^{n+1}$. 
Step II. Assume that $f$ is quasiconformal and compatible with $\Gamma$ on $R^{n}$. If there exist $p$ and $q \in \bar{R}^{n}$ such that $\Gamma \Lambda_{p q}$ is dense in $\mathrm{M}\left(R^{n}\right)$, and if at the same time $p$ is a nonsingular point of $f$, then $f$ is a Möbius transformation.

Here non-singular means that $f^{\prime}(p)$ exists and is $\neq 0$. It may be assumed that $(p ; q)=(0, \infty)$, for if $\Gamma$ and $f$ satisfy the original hypothesis, then $l_{p q} \Gamma l_{p q}^{-1}$ and $f \circ l_{p q}$ have the same property with respect to $(0, \infty)$. Even more obviously it is also legitimate to assume that $f(0)=0, f(\infty)=\infty$.

The gist of the argument is as follows: Because $\Gamma \Lambda_{0 \infty}$ is dense there exist sequences $\left\{\gamma_{i}\right\},\left\{\varrho_{i}\right\},\left\{k_{i}\right\}$ with $\gamma_{i} \in \Gamma, 0<\varrho_{i}<1, k_{i} \in \mathrm{O}(n)$ such that

$$
\gamma_{i} \varrho_{i} k_{i} \rightarrow I \text {. }
$$

On the other hand, because $f$ is compatible with $\Gamma$ there exist $\beta_{i} \in \Gamma$ with

$$
f \gamma_{i}=\beta_{i} f .
$$

By compactness of $\mathrm{O}(n)$ we may pass to a subsequence with $k_{i} \rightarrow k_{0}$, and the discreteness of $\Gamma$ implies $\varrho_{i} \rightarrow 0$.

From (5.3) and (5.4) one obtains

$$
\beta_{i}\left[f\left(\varrho_{i} k_{i}(x)\right)\right] \rightarrow f(x)
$$

and, in particular, $p_{i}=\beta_{i}(0) \rightarrow 0, q_{i}=\beta_{i}(\infty) \rightarrow \infty$. Since $l_{p_{i} q_{i}} \beta_{i}$ fixes 0 and $\infty$ one has $l_{p_{i} q_{i}} \beta_{i}=\lambda_{i} h_{i}$ for some $\lambda_{i}>0$ and $h_{i} \in \mathrm{O}(n)$. We may assume that $h_{i} \rightarrow h_{0}$. The fact that $l_{p_{i} q_{i}} \rightarrow I$ implies

$$
\beta_{i} \lambda_{i}^{-1} \rightarrow h_{0}
$$

The mappings $f_{i}(x)=\lambda_{i} f\left(\varrho_{i} x\right)$ are quasiconformal with bounded maximal dilatation. By a well known theorem [Vä] there is a subsequence of the $f_{i}$ which converges, uniformly on compact subsets of $R^{n} \backslash\{0\}$, to a limit function $\varphi$ which is either a quasiconformal mapping or a constant.

For this subsequence it follows from (5.5) and (5.6) that

$$
f(x)=\lim \beta_{i}\left[f\left(\varrho_{i} k_{0} x\right)\right]=\lim h_{o}\left[\lambda_{i}\left(f\left(\varrho_{i} k_{0} x\right)\right)\right]=h_{0}\left(\varphi\left(k_{0} x\right)\right)
$$

which shows, first of all, that $\varphi$ is not constant. Moreover, because $f^{\prime}(0)$ exists and is $\neq 0$, we see from

$$
\varphi(x)=\lim \lambda_{i} \varrho_{i} \frac{f\left(\varrho_{i} x\right)}{\varrho_{i}}
$$

that $\lim \lambda_{i} \varrho_{i}=\lambda$ exists and $\varphi(x)=\lambda f^{\prime}(0) x$. Thus $\varphi$ is linear, and by (5.7) the same is true of $f$.

Step I is now applicable unless $\infty$ is a fixed point of all $\gamma \in \Gamma$, a situation that would be in conflict with the denseness requirement. Step II is thereby proved. I wish to point out that the proof I have sketched is, up to notation, an almost verbatim rendition of Agard's own. 


\section{Myrberg's theorem}

The stage is now set for an elementary proof of Mostow's theorem. Only one thing is still needed, namely to show the existence of points $p$ and $q$ with the properties required for Step II. A quasiconformal mapping has almost no critical points. Therefore, when $n>1$, it is sufficient to show that the set of points $p$ for which there is a $q$ with $\Gamma \Lambda_{p q}$ dense in $\mathrm{M}\left(R^{n}\right)$ has positive measure, from which it follows by invariance that it has full measure when carried to $B^{n}$.

It is for this purpose that Agard has resuscitated and improved Myrberg's theorem. We give it the following setting:

Myrberg-Agard's Theorem. Let $\Gamma$ be a discrete subgroup of $\mathrm{M}\left(B^{n}\right), n>1$, of divergence type. Then, for a.e. $q \in S^{n-1}$ and for any choice of $p \in S^{n-1} \backslash\{q\}$ and a geodesic $l(s, t)$ there exists a sequence $\left\{\gamma_{i}\right\} \in \Gamma$ such that the images $\gamma_{i} l(p, q)$ converge to $l(s, t)$ while at the same time $\gamma_{i}^{\prime}(p) \rightarrow 0$.

Agard's proof is elementary, but long. It is modelled on Thurston's proof of the fact, discovered by Sullivan, that the conical limit set of a group of divergence type has full measure (for a detailed proof see [Ah 1]).

To show that $\Gamma \Lambda_{p q}$ is dense for a.e. $p$ and every $q$ we shall use Agard's own argument in spite of the fact that it involves an interchange of $p$ and $q$ which could have been avoided. Recall the definition (5.1) of $l_{p q}$ and observe that $l_{p q}^{\prime}(p)$ exists. Let $g \in \mathrm{M}\left(B^{n}\right)$, be arbitrary and choose $q, p$ as in the theorem as well as $s=g(p), t=g(q)$. Let $\left\{\gamma_{i}\right\}$ be the sequence whose existence is guaranteed by the theorem, and write $s_{i}=\gamma_{i}(p), t_{i}=\gamma_{i}(q)$ so that $s_{i} \rightarrow s, t_{i} \rightarrow t$ and $\gamma_{i}^{\prime}(0) \rightarrow 0$.

The transformations $l_{s_{i} t_{i}} \gamma_{i} l_{p q}^{-1}$ and $l_{s t} g l_{p q}^{-1}$ have 0 and $\infty$ as fixed points. Hence

$$
l_{s_{i} t_{i}} \gamma_{i} l_{p q}^{-1}=\lambda_{i} k_{i}, \quad l_{s t} g l_{p q}^{-1}=\lambda_{0} k_{0}
$$

with $\lambda_{i}, \lambda_{0}>0$ and $k_{i}, k_{0} \in O(n)$. Differentiation of the first equation at $x=0$ yields

$$
\lambda_{i} k_{i}=l_{s_{i} t_{i}}^{\prime}\left(s_{i}\right) \gamma_{i}^{\prime}(p) l_{p q}^{\prime}(p)^{-1}
$$

Because $l_{s_{i} t_{i}}^{\prime}\left(s_{i}\right) \rightarrow l_{s t}^{\prime}(s)$ and $\gamma_{i}^{\prime}(p) \rightarrow 0$ it follows that $\lambda_{i} \rightarrow 0$.

On the other hand, (6.1) also implies

$$
g=l_{s t}^{-1} \lambda_{0} k_{0} l_{p q}=\lim l_{s_{i} t_{i}}^{-1} \lambda_{0} k_{0} l_{p q}=\lim \gamma_{i} l_{p q}^{-1}\left(\lambda_{i}^{-1} \lambda_{0} k_{i}^{-1} k_{0}\right) l_{p q}
$$

where we have used the fact that $\lambda_{i}^{-1}$ and $\lambda_{0}$ commute with $k_{i}^{-1}$ and $k_{0}$. As soon as $\lambda_{i}<\lambda_{0}$ the multiplier $\lambda_{i}^{-1} \lambda_{0}$ in $l_{p q}^{-1}\left(\lambda_{i}^{-1} \lambda_{0} k_{i}^{-1} k_{0}\right) l_{p q}$ is $>1$ and we conclude that this factor belongs to $\Lambda_{q p}$. We read off from (6.3) that the arbitrary transformation $g \in \mathrm{M}\left(B^{n}\right)$ is in the closure of $\Gamma \Lambda_{q p}$. With a change of notation this shows that Step II is applicable, and the proof of Mostow's theorem is complete. 


\section{The use of Clifford numbers}

7.1. In a Comptes Rendus note of $1926 \mathrm{R}$. Fueter [Fu] showed that the transition from $\mathrm{M}(C)$ to $\mathrm{M}\left(U^{3}\right)$ can be easily and elegantly expressed in terms of quaternions. It seemed odd that this discovery should come so late, when quaternions were already quite unpopular, and sure enough a search of the literature by D. Hejhal turned up a paper from 1902 by K. Th. Vahlen [Va] where the same thing had been done, not only with quaternions but more generally, in any dimension, with Clifford numbers. It is strange that this paper passed almost unnoticed except for an unfavorable mention in an encyclopedia article by E. Cartan and E. Study. Vahlen was finally vindicated in 1949 when H. Maass [Ma] rediscovered and used his paper. Meanwhile the theory of Clifford algebras had taken a different course due to applications in modern physics, and Vahlen was again forgotten.

I am indebted to the Finnish mathematician P. Lounesto for having made me aware that there are two different ways in which Clifford numbers can be applied to Möbius groups. One is more general in that it applies to the conformal group of orthogonal spaces whose metric does not need to be positive definite, and there are several recent papers on this topic, including one by Lounesto and Latvamaa [LoLa]. Vahlen's method is more classical in that it uses two by two matrices, although with complex numbers replaced by certain Clifford numbers. Without prejudice in either direction, this is the method I shall talk about.

True to my background in complex analysis I shall follow Clifford's original definition and notation. The Clifford algebra $C_{n}$ shall be the associative algebra over the reals generated by elements $i_{1}, \ldots, i_{n-1}$ subject to the relations $i_{h} i_{k}=-i_{k} i_{h}$ for $h \neq k, i_{h}^{2}=-1$, and no others. $C_{1}$ can be identified with $R, C_{2}$ with $C$, and $C_{3}$ with the quaternion algebra $H$. In the last case $i, j, k$ are represented by $i_{1}, i_{2}$ and $i_{1} i_{2}$.

Each element $a \in C_{n}$ has a unique representation in the form $a=\sum a_{I} I$ where $a_{I} \in R$ and the summation is over all products $I=i_{h_{1}} i_{h_{2}} \ldots i_{h_{p}}$ with $0<h_{1}<\ldots<h_{p}<n$. The empty product $\emptyset$ is included and identified with the real number 1 . The coefficient of the empty product is denoted by $a_{0}$ and referred to as the real part $\operatorname{Re} a$.

$C_{n}$ is a vector space of real dimension $2^{n-1}$. The Clifford numbers of the special form $x=x_{0}+x_{1} i_{1}+\ldots+x_{n-1} i_{n-1}$ are called vectors. They form an $n$-dimensional subspace $V^{n}$ which we shall identify with $R^{n}$. In the newer literature it is more common to single out the space $X$ spanned by $i_{1}, \ldots, i_{n-1}$, but we follow the choice of Vahlen and Maass which is in better agreement with the complex case.

7.2. There are several involutions in $C_{n}$, similar to complex conjugation. The main involution consists in replacing every $i_{h}$ by $-i_{h}$. The image of $a$ under the main involution will be denoted by $a^{\prime}$. It is an automorphism in the sense that $(a+b)^{\prime}=$ $a^{\prime}+b^{\prime},(a b)^{\prime}=a^{\prime} b^{\prime}$. The second involution $a \rightarrow a^{*}$ is obtained by reversing the order of the factors in each $I=i_{h_{1}} \ldots i_{h_{p}}$. It is an anti-isomorphism satisfying $(a b)^{*}=b^{*} a^{*}$. These involutions can be combined to a third $a \rightarrow \bar{a}=a^{*}=a^{* \prime}$.

All three involutions map $V^{n}$ on itself. In particular $x^{*}=x$ for $x \in V^{n}$; unfortu- 
nately this disagrees with our earlier use of the star, but the notation $a^{*}$ chosen by Maass is so convenient that I would not change it. For vectors $x^{\prime}=\bar{x}$; I tend to prefer $\bar{x}$ because of the complex case. One verifies that $x \bar{x}=x_{0}^{2}+\ldots+x_{2-1}^{n}=|x|^{2}$ so that $|x|$ is the square norm. For two vectors $x \bar{y}+y \bar{x}=2(x, y)$. The notion of square norm carries over to all Clifford numbers: if $a=\sum a_{I} I$ we write $|a|^{2}=\sum a_{I}^{2}$, but it is not always true that $|a|^{2}=a \bar{a}$.

The center of $C_{n}$ will be denoted by $3_{n}$. By definition $a$ is in the center if it commutes with all Clifford numbers, and this will be so as soon as it commutes with $i_{1}, \ldots$, $i_{n-1}$. One shows easily that $3_{n}=R$ if $n$ is odd, but $3_{n}=R+R i_{1} \ldots i_{n-1}$ if $n$ is even.

7.3. Every non-zero vector $x$ is invertible with $x^{-1}=|x|^{-2} \bar{x}$. The product of invertible elements is invertible, hence every product of vectors is invertible. These products form a multiplicative group $\Gamma_{n}$ known as the Clifford group. The numbers in $\Gamma_{n}$ are the "good" Clifford numbers. It must always be kept in mind that the sum of two good numbers need not be good. If $a \in \Gamma_{n}$, then it is true that $|a|^{2}=a \bar{a}=\bar{a} a$, and if $a, b \in \Gamma_{n}$ then $|a b|=|a||b|$.

There is a more intrinsic characterization of the Clifford group: $a \in \Gamma_{n}$ in and only if $a$ is invertible and $a V^{n} a^{\prime-1}=V^{n}$. In other words, if $x$ is a vector so is $a x a,^{-1}$. The mapping $x \rightarrow a x a^{-1}$ is in fact a euclidean isometry, i.e. $a x a^{\prime-1}=\varrho(a) x$ where $\varrho(a) \in \mathrm{O}(n)$. This way of expressing rotations through Clifford numbers seems to be due to Lipschitz.

The following simple observation is in frequent use: If $a, b \in \Gamma_{n}$, then $a b^{-1}$ and $a^{*} b$ are simultaneously in $V^{n}$.

7.4. After these preliminaries I am ready to pass to the connection between Möbius transformations and Clifford numbers. As far as I can tell it is Vahlen's original idea to imitate the complex case and express an arbitrary Möbius transformation through a two by two matrix $\left(\begin{array}{ll}a & b \\ c & d\end{array}\right)$ whose entries are Clifford numbers. It is rather clear that arbitrary Clifford numbers will not do, and he imposes the a priori condition that the coefficients $a, b, c, d$ be either zero or in the Clifford group.

The matrix $g=\left(\begin{array}{ll}a & b \\ c & d\end{array}\right)$ is made to act on vectors $x \in V^{n}$ (or $R^{n}$ ) according to the rule

$$
g x=(a x+b)(c x+d)^{-1} .
$$

Strictly speaking this would require $c x+d$ to be invertible, but one can avoid this initial difficulty by declaring that $y=(a x+b)(c x+d)^{-1}$ shall mean the same as $y(c x+d)=a x+b$.

We would like $g$ to induce a mapping $\bar{V}^{n} \rightarrow \bar{V}^{n}$ where $\bar{V}^{n}=V^{n} \cup\{\infty\}$ with $\infty$ playing the same role as in $\bar{R}^{n}$. In other words, if $x$ is a vector or $\infty$ the same should be true of $g x$.

When does $g$ induce the identity mapping? This means that $a x+b=x(c x+d)$ for all $x$. For $x=0, \infty, 1$ it follows that $b=c=0, a=d$ and hence $a x=x a$ for all $x$. 
In other words $a \in 3_{n}$ and we see that the mapping (7.1) is not changed if $g$ is multiplied with any $a \in 3_{n} \backslash\{0\}$.

The immediate task is to find necessary and sufficient conditions under which $g$ induces a bijective mapping $\bar{V}^{n} \rightarrow \bar{V}^{n}$. We remark first that if $g_{1}$ and $g_{2}$ have this property, so does the matrix product $g_{1} g_{2}$ formed with attention to the non-commutativity. Secondly, the inverse mapping can also be expressed in the form (7.1). In fact, if $y(c x+d)=a x+b$ then $(a-y c) x=y d-b$. Because $x=x^{*}$ and $y=y^{*}$ this is the same as $x\left(-c^{*} y+a^{*}\right)=d^{*} y-b^{*}$ which is of the desired form. It follows that the matrices $g$ which induce bijections form a group, although we have not yet shown that their elements are in $\Gamma_{n} \cup\{0\}$. We know nevertheless that the matrices $\left(\begin{array}{ll}a & b \\ c & d\end{array}\right)$ and $\left(\begin{array}{rr}d^{*} & -b^{*} \\ -c^{*} & a^{*}\end{array}\right)$ induce mappings inverse to each other. Therefore their product, in either order, induces the identity. This implies the relations $c^{*} a=a^{*} c, b^{*} d=d^{*} b$, $a b^{*}=b a^{*}, c d^{*}=d c^{*}$ as well as $\Delta=a d^{*}-b c^{*} \in 3_{n}, \Delta_{1}=d^{*} a-b^{*} c \in 3_{n} . \Delta$ is the pseudodeterminant of $\left(\begin{array}{ll}a & b \\ c & d\end{array}\right)$.

Actually, the conditions $c^{*} a=a^{*} c$ etc. are consequences of the fact that $g 0$, $g \infty, g^{-1} 0, g^{-1} \infty$ are all in $V^{n}$. For instance, $g=a c^{-1} \in V^{n}$ implies $c^{*} a \in V^{n}$ and hence $c^{*} a=a^{*} c$; similarly for the others. We note further that $\Delta$ and $\Delta_{1}$ are $\neq 0$. This is so because $a d^{*}-b c^{*}=0$ would give $b^{-1} a=c^{*} d^{*-1}=\left(d^{-1} c\right)^{*}=d^{-1} c$ or $g^{-1} 0=g^{-1} \infty$, a contradiction; the case where $b$ or $d=0$ is easily disposed of.

At this point we can also fill in the missing information that $g_{1} g_{2}$ has entries in $\Gamma_{n} \cup\{0\}$ whenever $g_{1}, g_{2}$ induce bijective mappings and have their entries in the same set. This becomes clear on writing, for instance, $a_{1} a_{2}+b_{1} c_{2}=b_{1}\left(b_{1}^{-1} a_{1}+c_{2} a_{2}^{-1}\right) a_{2}$ where the middle factor is a sum of two vectors: the case when $b_{1}$ or $a_{2}=0$ is trivial. The other coefficients of $g_{1} g_{2}$ can be treated in the same manner.

Perhaps the most interesting observation is that $\Delta$ and $\Delta_{1}$ are not only in $3_{n}$, but actually real, except in the complex case $n=2$ where the determinant can of course be complex. Since there is nothing to prove when $n$ is odd it is sufficient to consider the case $n \geqq 4$, If $c$ and $d$ are both $\neq \neq 0$ one can write $\Delta=a c^{-1} \cdot c d^{*}-b d^{-1} \cdot d c^{*}$. Each term is the product of two vectors and cannot contain any term with $i_{1} \ldots i_{n-1} ; \Delta$ must therefore be real. If $c=0$ the image of $x=1$ is $(a 1+b) d^{-1} \in V^{n}$ and hence $a d^{-1} \in V^{n}$ and $\Delta_{1}=d^{*} a \in V^{n}$. This implies $d^{*} a \in R$ and also $\Delta=a d^{*} \in R$. The case $d=0$ is similar. Thus $\Delta$ and $\Delta_{1}$ are always real except for $n=2$.

7.5. The necessary conditions given above are not independent. One shows, for instance, that $a b^{*}$ and $c d^{*} \in V^{n}$ together with $\Delta \in R$ imply $a^{*} c, b^{*} d \in V^{n}$ and $\Delta_{1}=\Delta$. As a sample, $|d|^{2}=d^{*}\left(a d^{*}-b c^{*}\right) d^{\prime}=d^{*} a|d|^{2}-b^{*} d c^{*} d^{\prime}=\left(d^{*} a-b^{*} c\right)|d|^{2}$ and hence $\Delta=\Delta_{1}$. Similarly, $\bar{\Delta} c^{*} a=c^{*}\left(d^{\prime} \bar{a}-c^{\prime} \bar{b}\right) a=|a|^{2}(\bar{c} d)^{\prime}-|c|^{2} \bar{b} a \in V^{n}$.

The main result is that these conditions are also sufficient. Vahlen does not quite say so, but he essentially proves it. Maass is more explicit and more readable, fortyseven years later. Nevertheless, I would say that Vahlen has all the ingredients for the following theorem which I state in three parts: 
Theorem.

I. $x \rightarrow g x=(a x+b)(c x+d)^{-1}$ with $a, b, c, d \in \Gamma_{n} \cup\{0\}$ is a bijective mapping $\bar{V}^{n} \rightarrow \bar{V}^{n}$ if and only if $a c^{*}, b d^{*} \in V^{n}$ and $\Delta(g)=a d^{*}-b c^{*} \in R \cup\{0\}$.

II. This mapping extends to a bijection $\bar{V}^{n+1} \rightarrow \bar{V}^{n+1}$ by adjoining $i_{n}$ to $C_{n}$. If $\Delta(g)>0$ the upper half-space $U^{n+1}$ is mapped on itself.

III. All the mappings are conformal, hence given by Möbius transformations in $\mathrm{M}\left(R^{n}\right)$ and $\mathrm{M}\left(U^{n+1}\right)$, respectively. Conversely, every sense-preserving Möbius transformation can be expressed in the form (7.1) with $\Delta(g)>0$.

A few comments are in order. First of all, one can always normalize to $\Delta(g)=1$ or -1 . Maass postulates $\Delta(g)=1$ and is not aware that $\Delta(g)$ has to be real; neither was I until quite recently. The passage from I to II is obvious from $\bar{V}^{n} \subset \bar{V}^{n+1}$ and $\Gamma_{n} \subset \Gamma_{n+1}$. The second part of III is almost obvious, for it is sufficient to express the generators through matrices. The important part of the theorem, as I see it, is that it gives an explicit rule for determining whether a given matrix $\left(\begin{array}{ll}a & b \\ c & d\end{array}\right)$ does or does not induce a Möbius transformation.

As for the proof, practically everything follows from the identity

$$
g x-(g y)^{*}=\Delta(g)\left(y c^{*}+d^{*}\right)^{-1}(x-y)(c x+d)^{-1}
$$

which is a direct consequence of $a^{*} c=c^{*} a, b^{*} d=d^{*} b$ and $\Delta_{1}=\Delta \in R$. It implies $(g y)^{*}=g y$ so that the left hand side of (7.2) can be replaced by $g x-g y$. If applied with $y=0$ one obtains, after passing to the inverses,

$$
(g x-g 0)^{-1}=\Delta(g)^{-1}\left(c d^{*}+d x^{-1} d^{*}\right)
$$

which does indeed show that $(g x-g 0)^{-1}, g x-g 0$ and $g x$ itself are vectors, thereby proving the sufficiency. For more details I refer to a forthcoming article [Ah 2].

\section{References}

[Ag 1] AgARd, S.: Elementary properties of Möbius transformations in $R^{n}$ with applications to rigidity theory. - University of Minnesota Report, 82-110.

[Ag 2] AGARD, S.: A geometric proof of Mostow's rigidity theorem for groups of divergence type. Acta Math. (to appear).

[Ah 1] Ahlfors, L. V.: Möbius transformations in several dimensions. - Lecture Notes, University of Minnesota, 1981.

[Ah 2] Ahlfors, L. V.: Möbius transformations and Clifford numbers. - To appear.

[Be] Beardon, A. F.: The geometry of discrete groups. - Springer-Verlag, Berlin-HeidelbergNew York, 1983.

[Fu] Fueter, R.: Sur les groupes improprement discontinus. - C. R. Acad. Sci. Paris 182, 1926, $432-434$.

[Ku] Kuusalo, T.: Boundary mappings of geometric isomorphisms of Fuchsian groups. - Ann. Acad. Sci. Fenn. Ser. A. I. Math. 545, 1973, 1-7. 
[Lola] Lounesto, P., and E. LatvamaA: Conformal transformations and Clifford algebras. - Proc. Amer. Math. Soc. 79: 4, 1980, 533-538.

[Ma] MaAss, H.: Automorphe Funktionen von mehreren Veränderlichen und Dirichletsche Reihen. - Abh. Math. Sem. Univ. Hamburg 16, 1949, 72-100.

[Mo] Mostow, G. D.: Quasi-conformal mappings in $n$-space and the rigidity of hyperbolic space forms. - Inst. Hautes Études Sci. Publ. Math. 34, 1968, 53-104.

[My] Myrberg, P. J.: Ein Approximationssatz für die Fuchsschen Gruppen. - Acta Math. 57, 1931, 389-409.

[Su] Sullivan, D.: On the ergodic theory at infinity of an arbitrary discrete group of hyperbolic motions. - Riemann surfaces and related topics: Proceedings of the 1978 Stony Brook Conference, edited by I. Kra and B. Maskit, Annals of Mathematics Studies 97, Princeton University Press, Princeton, New Jersey, 1981, 465-496.

[Ts] Tsus, M.: Potential theory in modern function theory. - Maruzen Co., Tokyo, 1959.

[Tu] TUKIA, P.: Rigidity theorems for Möbius groups. - To appear.

[Va] Vahlen, K. TH.: Ueber Bewegungen und complexe Zahlen. - Math. Ann. 55, 1902, 585593.

[Vä] VÄISÄLÄ, J.: Lectures on $n$-dimensional quasiconformal mappings. - Lecture Notes in Mathematics 229, Springer-Verlag, Berlin-Heidelberg-New York, 1971.

[Wi] Wielenberg, N. J.: Discrete Möbius groups: fundamental polyhedra and convergence. Amer. Journal, Vol. 99, No. 4, 861-877.

\section{Harvard University}

Department of Mathematics

Cambridge, Mass. 02138

USA

Received 2 December 1983 